\title{
Sperm Patch-Clamp
}

\author{
Polina Lishko*,1, David E. Clapham ${ }^{\dagger, \ddagger, 1}$, Betsy Navarro ${ }^{\dagger, \ddagger}$, and Yuriy Kirichok ${ }^{\S}$ \\ "Department of Molecular and Cell Biology, University of California, Berkeley, Berkeley, \\ California, USA \\ †Department of Neurobiology, Harvard Medical School, Boston, Massachusetts, USA \\ $\neq$ Department of Cardiology, Howard Hughes Medical Institute, Manton Center for Orphan \\ Disease, Children's Hospital, Boston, Massachusetts, USA \\ $\S$ Department of Physiology, University of California, San Francisco, San Francisco, California, \\ USA
}

\begin{abstract}
Sperm intracellular $\mathrm{pH}$ and calcium concentration $\left(\left[\mathrm{Ca}^{2+}\right]_{i}\right)$ are two central factors that control sperm activity within the female reproductive tract. As such, the ion channels of the sperm plasma membrane that alter intracellular sperm $\left[\mathrm{Ca}^{2+}\right]$ and $\mathrm{pH}$ play important roles in sperm physiology and the process of fertilization. Indeed, sperm ion channels regulate sperm motility, control sperm chemotaxis toward the egg in some species, and may trigger the acrosome reaction. Until recently, our understanding of these important molecules was rudimentary due to the inability to patchclamp spermatozoa and directly record the activity of these ion channels under voltage clamp. Recently, we overcame this technical barrier and developed a method for reproducible application of the patch-clamp technique to mouse and human spermatozoa. This chapter covers important aspects of application of the patch-clamp technique to spermatozoa, such as selection of the electrophysiological equipment, isolation of spermatozoa for patch-clamp experiments, formation of the gigaohm seal with spermatozoa, and transition into the whole-cell mode of recording. We also discuss potential pitfalls in application of the patch-clamp technique to flagellar ion channels.
\end{abstract}

\section{INTRODUCTION}

Sperm ion channels play a central role in the regulation of sperm cell ion homeostasis and vital cellular processes such as motility, chemotaxis toward the egg, and the acrosome reaction. Mutation or absence of some ion channels directly affects male fertility of both mice and humans (Darszon, Nishigaki, Beltran, \& Trevino, 2011; Hildebrand et al., 2010; Kirichok \& Lishko, 2011; Lishko et al., 2012; Ren \& Xia, 2010). Mature mammalian spermatozoa are stored in a quiescent state in the caudal portion of the epididymis and vas deference. They acquire progressive motility for the first time upon ejaculation when mixed with the seminal plasma. However, these motile spermatozoa are still unable to fertilize the egg. They acquire their fertilizing ability only after several hours of exposure to fluids of the female reproductive tract. This final functional maturation is called sperm capacitation.

Some experimental evidence suggests that mammalian spermatozoa use chemotaxis to find the egg in the labyrinth of epithelial folds of the Fallopian tubes (Eisenbach \& Giojalas, 2006; Kaupp, Kashikar, \& Weyand, 2008). In the ascent of the female reproductive tract, spermatozoa develop hyper-activation to free themselves from impediments and eventually

(C) 2013 Elsevier Inc. All rights reserved.

${ }^{1}$ Corresponding authors: lishko@berkeley.edu; dclapham@enders.tch.harvard.edu. 
penetrate the cumulus oophorus and the zona pellucida, the protective vestments on the egg's surface (Suarez \& Pacey, 2006). Hyperactivation is characterized by an asymmetrical, high-amplitude beat of the sperm flagellum (Suarez, 2008) that generates higher motile force than normal swimming motions (low-amplitude symmetrical flagellar beat). Finally, for penetration through the egg's protective vestments, hyperactivation is complemented by the release of the hydrolytic enzymes from the tip of the sperm's head upon exocytosis of the acrosomevesicle(theacrosome reaction)(Dean, 2007; Florman, Jungnickel, \& Sutton, 2008; Wassarman \& Litscher, 2008). All these events eventually result in the fusion between the egg and the sperm cells and the conception of a new life.

Interestingly, sperm motility activation, capacitation, sperm chemotaxis, hyperactivation, and the acrosome reaction are triggered by elevation of the intracellular $\mathrm{pH}$ and $\left[\mathrm{Ca}^{2+}\right]$ in all or specific domains of the sperm cell (Kirichok \& Lishko, 2011; Lishko et al., 2012; Publicover, Harper, \& Barratt, 2007). These changes in intracellular $\mathrm{pH}$ and $\left[\mathrm{Ca}^{2+}\right]$ are caused by ion channels and transporters of the sperm plasma membrane that allow $\mathrm{H}^{+}$to leave cytoplasm, conduct $\mathrm{Ca}^{2+}$ into the cell, and control the membrane potential to regulate ion transport of the sperm plasma membrane. To stress the significance of ion channels on sperm physiology, we assert that ion channels and transmembrane ion transport are as important for spermatozoa as they are important for electrical signaling in neurons. At this time, several different ion channels have been convincingly demonstrated to function in spermatozoa.

The CatSper channel comprises four different pore-forming subunits (CatSper1-CatSper 4) and at least three auxiliary subunits (CatSper $\beta$, Cat-Sper $\gamma$, and CatSper $\delta$ ) and is the principal $\mathrm{Ca}^{2+}$ channel of the sperm plasma membrane (Chung, Navarro, Krapivinsky, Krapivinsky, \& Clapham, 2011; Kirichok, Navarro, \& Clapham, 2006; Liu, Xia, Cho, Clapham, \& Ren, 2007; Quill, Ren, Clapham, \& Garbers, 2001; Ren et al., 2001; Wang, Liu, Cho, \& Ren, 2009). The CatSper channel is primarily responsible for elevation of intracellular $\left[\mathrm{Ca}^{2+}\right]$, controls sperm hyperactivation, and may affect various other $\mathrm{Ca}^{2+}$ dependent sperm responses such as chemotaxis and the acrosome reaction (Carlson et al., 2005, 2003; Suarez, 2008). The CatSper channel is activated by intracellular alkalinization (in mice and humans) and progesterone (humans) (Kirichok et al., 2006; Lishko, Botchkina, Fedorenko, \& Kirichok, 2010; Lishko, Botchkina, \& Kirichok, 2011). Mice and humans lacking CatSper channels are infertile.

Powered by glycolysis and oxidative phosphorylation during high motility, the sperm flagellum quickly acidifies. The removal of protons from the sperm flagellum is an essential process for maintaining motility - the axoneme is unable to function effectively below $\mathrm{pH}$ 6.0. To expel protons and support motility, spermatozoa possess specialized proton channels and/or transporters. Human sperm plasma membrane has a robust $\mathrm{H}^{+}$conductance HSper that appears to be mediated by the voltage-gated $\mathrm{H}^{+}$channel (Hv1) (Lishko et al., 2010; Lishko \& Kirichok, 2010). HSper is likely to be responsible for rapidly alkalinizing sperm; it is activated by membrane depolarization, removal of extracellular zinc, and anandamide (Lishko et al., 2010). Interestingly, HSper currents cannot be detected in mouse spermatozoa, suggesting a different mechanism for sperm intracellular alkalinization in mice (Lishko et al., 2010).

The KSper channel, formed as a tetramer of Slo3 proteins, is the principal $\mathrm{K}^{+}$channel in mouse and perhaps human sperm (Navarro, Kirichok, Chung, \& Clapham, 2008; Navarro, Kirichok, \& Clapham, 2007; Santi et al., 2010; Zeng, Yang, Kim, Lingle, \& Xia, 2011). KSper sets the sperm membrane potential and thus regulates the activity of other voltagesensitive sperm ion channels (Navarro et al., 2007; Zeng et al., 2011) such as CatSper and Hv1. Along with KSper, other channels may also affect sperm membrane potential. Mouse 
spermatozoa also possess a nonselective cationic ATP-activated P2X2 channel confined to the sperm midpiece (Navarro, Miki, \& Clapham, 2011). In addition, $\mathrm{a} \mathrm{Cl}^{-}$channel activated by intracellular $\mathrm{Ca}^{2+}$ (likely mediated by TMEM16A) has recently been identified in human spermatozoa (Orta et al., 2012). Various other channels have been suggested to play a role in sperm physiology, but it remains to be established if these channels are indeed present and functionally active in mature spermatozoa (Darszon et al., 2006; Meizel, 2004). It should also be noted that sperm ion channels and their regulation can differ significantly between species (Kirichok \& Lishko, 2011).

The gold standard of ion channel measurement is control of membrane voltage and measurement of current response (voltage clamp). Until recently, voltage clamp of whole spermatozoan membranes had not been achieved due to the difficulty of obtaining gigaseals along most to the cell's membrane. Spermatozoa are highly motile, elongated cells with micron cross sections. However, the main barrier for the sperm patch clamp was extremely low volume of the sperm cytoplasm and tight attachment of the sperm plasma membrane to rigid intracellular structures (Kirichok \& Lishko, 2011). These morphological properties made it impossible to pull enough sperm plasma membrane inside the recording pipette to form a tight seal between the sperm plasma membrane and the glass patch pipette, a prerequisite for successful patch-clamp recording (Fig. 4.1A). Even indirect, optical methods to study sperm ion channels with fluorescent $\mathrm{Ca}^{2+}$ and $\mathrm{H}^{+}$indicators were prone to error due to the extremely low cell volume and a weak signal originating from a single cell (Darszon et al., 2004; Kirichok \& Lishko, 2011).

Recently we demonstrated that despite the technical difficulties, the patch-clamp technique can be successfully applied to mouse and human spermatozoa (Kirichok et al., 2006; Lishko et al., 2010) (Figs. 4.2 and 4.3). This advance has been made possible by identification of the region of the sperm plasma membrane (the cytoplasmic droplet, $\mathrm{CD}$ ) that is loosely attached to the rigid intracellular structures and with which a tight gigaohm seal can be formed with the patch pipette (Figs. 4.1B-D, 4.2, and 4.3) (Kirichok \& Lishko, 2011; Kirichok et al., 2006; Lishko et al., 2010). In only a few years since its development, the sperm patch-clamp technique has resulted in a tremendous progress in our understanding of the sperm ion channels.

Interestingly, the majority of ion channels to date are located to the sperm flagellum (Kirichok \& Lishko, 2011; Lishko et al., 2012). This is not surprising, given the fact that the membrane of the sperm flagellum constitutes about $70-75 \%$ of the total sperm plasma membrane. Furthermore, for both mouse and human spermatozoa, it is possible to disassociate the flagellum (the principle piece plus the midpiece) from the head and record currents from the whole flagellar plasma membrane only (Kirichok \& Lishko, 2011; Kirichok et al., 2006; Lishko et al., 2011). Thus, the sperm patch-clamp technique can be used to study flagellar ion channels and the mechanisms that regulate their activity to provide important insights into the mechanisms of membrane-associated signal transduction in ciliary structures. Below we present detailed protocols for application of the patch-clamp technique to mouse and human spermatozoa.

\section{EQUIPMENT}

The selection of equipment for sperm patch clamp is dictated by the small size of the sperm $\mathrm{CD}$ to which the glass micropipette has to be attached to form the gigaohm seal. The usual size of the CD in mouse and human spermatozoa is around $2 \mu \mathrm{m}$, and application of the patch-clamp technique to such a small object requires high-resolution optics. Our patchclamp rigs are equipped with inverted Olympus IX71 microscope with differential interference contrast (DIC) optical components. Using a microscope equipped with DIC 
optics for successful sperm patch clamp is highly recommended over phase-contrast optics since the spatial information seen in DIC helps achieve superior precision in positioning a patch pipette onto the tiny $\mathrm{CD}$.

We use an Olympus UPlanSApo 60× water immersion objective, with numerical aperture of 1.20. This objective features long working distance $(0.28 \mathrm{~mm})$, which allows observation of free-swimming sperm cell in solution. The objective also has an adjustment collar for the thickness of the coverslip (variable from 0.13 to $0.21 \mathrm{~mm}$ ). This combination of the long working distance and the adjustment collar allows for observation through two $0.13-\mathrm{mm}$ coverslips (a coverslip serving as a glass bottom of the recording chamber plus a round 5$\mathrm{mm}$ coverslip with deposited sperm cells placed into the recording chamber). As will be discussed below, depositing sperm cells on easily exchangeable round 5-mm coverslips (rather than on the bottom of the recording chamber directly) is a convenient way to load fresh sperm cells into the recording chamber. In addition to the Olympus UPlanSApo 60x objective, our IX71 microscopes are equipped with 10x eyepieces and an optional 1.6x internal magnification. The regular internal magnification of the microscope $(1 \times)$ is used to locate the appropriate sperm cell to patch clamp, and when the patch pipette is near the selected sperm cell, the 1.6 internal magnification is engaged to increase magnification.

The small size of the $\mathrm{CD}$ also requires manufacturing of glass micropipettes with extremely fine tips. For pipette fabrication, we use Sutter P97 or P1000, micropipette puller, and Sutter borosilicate glass capillaries with outer diameter $1.5 \mathrm{~mm}$, inner diameter $0.86 \mathrm{~mm}$, and an internal filament. We fire-polish pipettes under 100× magnification (100/0.80 ELWD 210/0 objective) using a Narishige Micro Forge MF-830. Fire polishing is essential step for successful seal formation between the micropipette and the CD.

Sperm patch clamping requires a precise micromanipulator without drift. We use a Sutter MPC 385 micromanipulator that is attached to the Olympus IX71 microscope with a Sutter MD series micromanipulator platform. This platform is attached directly to the microscope and ensures better stability than an independent micromanipulator stand. The assembled rig is positioned on a vibration-damping air table (e.g., Newport). The absence of any vibration is essential part of a successful sperm patch-clamp recording since even a slight vibration will ultimately compromise achievement of a successful seal formation and the break-in. To test the setup, one should not see any movement of the pipette tip (under $60 \times$ magnification) even when a person jumps up and down near the table.

Finally, we use a Warner Instruments RC-24E perfusion chamber connected via a manifold to a gravity-fed perfusion system. The RC-24E is a low-volume diamond-shaped chamber with laminar flow and relatively fast solution exchange rates. A 5-mm coverslip with predeposited sperm cells fits perfectly into the diamond-shaped opening of this chamber. To perfuse a solution through the recording chamber, we use a simple home-made gravity-fed perfusion system with a Teflon manifold (MP-8, Warner Instruments, cat. \# 64-0211) that allows one to connect the perfusion chamber with up to eight separate perfusion lines. The tubing should not leach plasticizers into the solutions since such chemicals can significantly alter ion channel activity. We use small-diameter Teflon tubing as the main perfusion tubing. As Teflon tubing is rather stiff, flexible connections are made of high purity silicon tubing that fits over the Teflon tubing. In addition, we use syringes (Air Tite Norm-Ject brand) that lack any lubricant since the mineral oil or other lubricating additives can interfere with ion channel recording. 


\section{ISOLATION OF SPERMATOZOA FOR PATCH-CLAMP EXPERIMENTS}

\subsection{Selection of a suitable sperm developmental stage}

Human freshly ejaculated spermatozoa are considered mature and are used in sperm patchclamp experiments. Ejaculated spermatozoa not only have completed the process of the morphological and functional maturation in the testis and epididymis (Cornwall, 2009; Yanagimachi, 1994), but they have also been exposed to the seminal plasma and have undergone possible modifications by its proteins and other organic and inorganic components (Maxwell, de Graaf, Ghaoui Rel, \& Evans, 2007). Luckily, CDs are not shed from human sperm and some primate sperm during ejaculation (Cooper, 2011), and the patch-clamp technique can be applied to completely mature ejaculated human spermatozoa (Lishko et al., 2010). In other species, such as mouse, rabbit, and dogs, the droplet is normally shed (without disrupting the integrity of the plasma membrane) during ejaculation. Human spermatozoa preserve the droplet within the female reproductive tract, and it does not interfere with the process of fertilization (Cooper, 2011). Ejaculated human spermatozoa can also be in vitro capacitated, and the patch-clamp technique can be applied equally well to both capacitated and uncapacitated ejaculated human spermatozoa. However, capacitated spermatozoa swim more vigorously and therefore stick less often to the coverslip (see Section 4.2, step 3).

In contrast to human spermatozoa, collection of ejaculated spermatozoa in mice is difficult (Yamauchi \& Ward, 2007), and the sperm CD is often shed upon ejaculation (Cooper, 2011). Therefore, to understand ion channel physiology of mature mouse spermatozoa, patch-clamp experiments can be conducted with cells isolated from either corpus or cauda epididymis (Kirichok et al., 2006; Lishko et al., 2010, 2011; Navarro et al., 2007). Although they have not been exposed to the seminal plasma, mouse spermatozoa from the cauda epididymis are generally considered mature, and upon in vitro capacitation acquire the ability to fertilize the egg (Hoppe, 1975; Yanagimachi, 1994). Unfortunately, CDs of cauda spermatozoa are fragile (perhaps due to imminent shedding), which makes patch-clamp recording from cauda sperm more difficult, but possible. In contrast, mouse spermatozoa isolated from corpus epididymis have robust CDs and are easier to patch clamp. However, spermatozoa from corpus epididymis are not completely mature, and the majority of them are unable to fertilize the egg (Hoppe, 1975; Yanagimachi, 1994). In contrast to cauda spermatozoa, they also are less able to undergo capacitation, which limits their use as a model for understanding of this important process. Nevertheless, so far, we have not noticed any significant differences between ion channels in corpus and caudal mouse spermatozoa, and in many cases, caudal spermatozoa should be suitable for studies of ion channels in noncapacitated spermatozoa. Thus, mouse spermatozoa from cauda epididymis appear to be the best available system for understanding the ion channels properties of mature mouse sperm. Corpus spermatozoa are easier to patch clamp, and they can be used with great success in certain experiments, especially when the results are subsequently confirmed with a sufficient number of caudal spermatozoa.

In mice and humans, the $\mathrm{CD}$ is present on the sperm cell from the moment it is released from the germinal epithelium into the lumen of the seminiferous tubule until the final stage of its development in the cauda epididymis. Furthermore, mouse caudal and human ejaculated sperm can be capacitated, and any capacitation-associated changes in the properties of the sperm ion channel can be studied. Since in humans the CD is not shed during ejaculation, ion channel activity can, in principle, be compared between cauda epididymis and ejaculated spermatozoa in order to study the effect of exposure to the seminal plasma. Studies of testicular and epididymal spermatozoa in humans are significantly limited by the availability of postoperative material. 


\subsection{Isolation of epididymal mouse spermatozoa}

C57B1/6 male mice aged 3-8 months are euthanized by inhalation of $\mathrm{CO}_{2}$ followed by cervical dislocation. These methods are consistent with the recommendations of the Panel on Euthanasia of the American Veterinary Medical Association and IACUC Committee. Tissue collection (cauda or corpus epididymi) from mice is performed immediately after cervical dislocation as described:

1. The lower abdominal area is opened with scissors and both epididymides are extracted and washed in a 35-mm cell culture dish containing HS (HEPES saline) solution as follows: $135 \mathrm{~m} M \mathrm{NaCl}, 5 \mathrm{~m} M \mathrm{KCl}, 1 \mathrm{~m} M \mathrm{MgSO}_{4}, 2 \mathrm{~m} M \mathrm{CaCl}_{2}, 5 \mathrm{~m} M$ glucose, $1 \mathrm{~m} M$ sodium pyruvate, $10 \mathrm{~m} M$ lactose, and $20 \mathrm{~m} M$ HEPES, pH 7.4 (with $\mathrm{NaOH}$ ). HS should be kept at $4{ }^{\circ} \mathrm{C}$ (recommend $<1$ month). An aliquot of the HS solution should be warmed to room temperature before contact with sperm cells.

2. The epididymides are then transferred into a new cell culture dish containing the HS solution and thoroughly cleaned from of residual fat. They are then divided into caput, corpus, and cauda using a scalpel.

3. The corpus (or cauda, as needed) of each epididymis is transferred into a new cell culture dish containing HS solution and multiple incisions made in the isolated part of the epididymis using a pointed scalpel blade \#11.

4. The parts of the epididymis with multiple incisions are transferred into a 1.5-ml Eppendorf tube containing $1.5 \mathrm{ml}$ HS solution. Using super-fine Dumont type 5a forceps, sperm cells are briefly shaken from epididymis into the solution; epididymides are discarded and the tube is left to settle for $10 \mathrm{~min}$.

5. After the solid matter (non-sperm cells) sediments to the bottom of the Eppendorf tube, the supernatant is transferred into another 1.5-ml Eppendorf tube. This tube is centrifuged $(500 \mu \mathrm{g}, 5 \mathrm{~min})$ at room temperature.

6. Second wash step. The supernatant is removed, and the sperm cells are resuspended in fresh $1.5 \mathrm{ml} \mathrm{HS}$ solution. Centrifugation is repeated and the supernatant is removed.

7. The pellet is resuspended in $0.5 \mathrm{ml}$ of $\mathrm{HS}$ solution and stored at room temperature for up to $6 \mathrm{~h}$ in a 1.5-ml Eppendorf tube.

\subsection{Collection and isolation of ejaculated human spermatozoa}

All protocols and experiments with human spermatozoa have to be approved by the Committee on Human Research. Human specimens, such as blood and semen, are biohazards and therefore must be handled according to Biosafety Level II laboratory standards.

Sperm donors are requested to refrain from sexual activity for at least 2 days prior to the specimen collection to ensure the highest number of sperm cells. Semen specimens are usually collected at home and delivered to the laboratory within $1 \mathrm{~h}$. Donors are asked to collect the semen specimen by masturbation into a clean, dry, sterile container. We prefer to use poly-propylene (PP) collection cups, devoid of plasticizers and bisphenol A. Sarstedt (product number 75.9922.721; 70-ml container with assembled yellow lid, sterile, and individually wrapped); urine collection containers work well for our applications.

Ideally, the entire ejaculate should be collected. We ask our donors to avoid coitus interruptus as a mean of collection since it is possible that the first portion of the ejaculate, which usually contains the highest concentration of spermatozoa, will be lost. Lubricants, 
condoms, or condoms with spermicidal components or powders should not be used for semen collection.

After collection, the container is tightly closed and is labeled with the date and time of collection. The specimen has to be delivered to the laboratory within $1 \mathrm{~h}$ after collection. During transport, the specimen is protected from extreme temperatures and sunlight and should be kept at $25-36^{\circ} \mathrm{C}$. Upon delivery to the lab, spermatozoa are purified by the swimup technique as described below.

Before processing, freshly ejaculated semen specimens are allowed to undergo liquefaction, as otherwise semen coagulates upon contact with water-based solutions. During liquefaction, freshly ejaculated semen is simply allowed to remain at room temperature for 20-60 min. Liquefaction transforms semen, initially a thick hyperviscous gel, into a more liquid state. During this time, the gel formed by proteins of seminal vesicles (e.g., semenogelin) is degraded by seminal proteases. Liquefaction starts at the time of ejaculation. Thus if the specimen was collected at home and delivered to the lab, the delivery time is added to the total time of liquefaction. Ideally, semen should not be allowed to liquefy for more than $1 \mathrm{~h}$ since prolonged exposure to the seminal plasma can adversely affect spermatozoa.

After the liquefaction, spermatozoa are purified from semen by the swim-up method. The swim-up method does not use centrifugation and is gentler as compared to the more widely used spin-down procedure that can damage sperm membranes. Swim-up purification is performed in artificial human tubal fluid solution (HTF, in $\mathrm{m} M$ ): $98 \mathrm{NaCl}, 4.7 \mathrm{KCl}, 0.4$ $\mathrm{KH}_{2} \mathrm{PO}_{4}, 2 \mathrm{CaCl}_{2}, 0.2 \mathrm{MgSO}_{4}, 20$ HEPES, 3 glucose, 21 lactic acid, 0.3 sodium pyruvate, $\mathrm{pH} 7.4$ (adjusted with $\mathrm{NaOH}$ ). After preparation, $\mathrm{HTF}$ is kept at $4{ }^{\circ} \mathrm{C}$ for no more than 1 month. HTF should be warmed up to room temperature before contact with the semen. Isolation of human spermatozoa by the swim-up technique is performed as follows:

1. Add $4 \mathrm{ml}$ of HTF into a 50-ml PP BD Falcon tube; carefully underlay with $1 \mathrm{ml}$ of liquefied ejaculate. To handle the thick semen sample, we use a positive displacement pipette (Gilson Microman M1000 with CP1000ST Gilson tips). Repeat this step until all ejaculate is used. If the total amount of semen was $5 \mathrm{ml}$, then five Falcon tubes each containing $1 \mathrm{ml}$ of semen and $4 \mathrm{ml}$ of HTF are prepared. The tubes are placed in a stand that holds them at a $45^{\circ}$ angle in order to increase the swim-up surface.

2. The stand and tubes are placed into a $37{ }^{\circ} \mathrm{C}$ incubator for 1 h. $\mathrm{CO}_{2}$ is not used in this procedure. If placed in a $\mathrm{CO}_{2}$ incubator, close the cap tightly to prevent gas exchange and acidification of the HTF buffer.

3. After $1 \mathrm{~h}, 2-3$-ml upper fractions, containing only healthy motile spermatozoa, are carefully collected from each 50-ml tube and combined in a $15-\mathrm{ml}$ conical tube. Precautions are made not to pick up semen when collecting the upper fraction. On average, $\sim 2-5 \times 10^{7}$ sperm cells per ejaculate are collected. Cells are counted by using a hemocytometer or a counting chamber (Spectrum technologies, Chamber slide, SC 20-01-C) according to manufacturer's instructions.

4. The $15-\mathrm{ml}$ conical tube is left to stand vertically for $\sim 30-60 \mathrm{~min}$ at room temperature. Eventually, spermatozoa concentrate at the bottom (a thick white cloud will form) as they become dormant in HTF solution. However, spermatozoa resume their vigorous motility when placed in HS solution (see below). For patchclamp experiments, human spermatozoa are stored in HTF solution for up to $6 \mathrm{~h}$ at room temperature. 


\section{PATCH-CLAMP RECORDING}

\subsection{Difference in sperm patch-clamp recording from traditional recordings}

Sperm patch clamp varies in some details from the patch-clamp technique developed by Erwin Neher and Bert Sakmann (Hamill, Marty, Neher, Sakmann, \& Sigworth, 1981; Sakmann \& Neher, 1984). In the past, the principal difficulty with sperm patch clamp was an inability to form a gigaohm seal between the glass patch pipette and the sperm cell, regardless of species. The reason for this difficulty was not immediately clear; as for many cells, the plasma membrane easily forms a gigaohm seal with the glass pipette. The composition of the sperm plasma membrane and proteins associated with its outer leaflet was often blamed for the inability to form gigaohm seals with spermatozoa. However, we discovered that the principal reason for this is the tight association of the sperm plasma membrane with the underlying structures (Kirichok \& Lishko, 2011; Kirichok et al., 2006). The sperm plasma membrane, by itself, can form gigaseals with the patch pipette.

Spermatozoa have a low ratio of cytoplasm to plasma membrane due to their geometry. The sperm plasma membrane is tightly associated with rigid intracellular structures. These two structural features result in a slim, arrow-like cell that is designed to penetrate through highly viscous environments such as egg's protective vestments, without deformation or damage to the plasma membrane. Although the special architecture of the sperm cytosol and the plasma membrane is indispensible for the function of the sperm cell, it also makes sperm patch clamp extremely difficult. To form a gigaohm seal, negative pressure is created in the pipette to draw a small portion of the sperm plasma membrane into the tip of pipette. This portion of the membrane forms an $\Omega$-shaped invagination into the tip of the pipette and establishes a tight seal gigaohm seal with its internal walls (Fig. 4.1A). However, since the sperm plasma membrane is tightly attached to the rigid intracellular structures, spermatozoa have no "spare" plasma membrane to be drawn into the pipette to form the $\Omega$-shaped invagination, essential for gigaohm seal formation (Suchyna, Markin, \& Sachs, 2009), and therefore, the gigaohm seal cannot be formed.

We identified the sperm $\mathrm{CD}$ as the only portion of the sperm plasma membrane where the gigaohm seal with the patch pipette can be formed with current techniques. The $\mathrm{CD}$ is a small (normally 1-3 $\mu M$ in diameter) remnant of the spermatid's cytoplasm and can be found somewhere along the sperm midpiece (Figs. 4.1B-D and 4.2A). The CD is simply the remnant of the spermatozoan cell from which the head and tail develop-functionally, it may help adapt to changes in extracellular osmolarity during ejaculation. The plasma membrane within the $\mathrm{CD}$ is not attached to the rigid intracellular structures and can be drawn into the pipette to form a gigaohm seal. All our attempts to form a seal outside of CD have been unsuccessful despite thousands of attempts (Ren et al., 2001). Thus, although the physiological function of the sperm CD remains vague, its practical significance is obvious: it is the only "jack" on the sperm surface through which one can connect the patch-clamp amplifier.

\subsection{Recording from the whole sperm plasma membrane (both human and mouse)}

1. Glass micropipettes for whole-cell sperm patch-clamp recordings are pulled from borosilicate glass filaments (Sutter Instruments, cat. \# BF150-86-7.5) using, for example, a Sutter Flaming/Brown Micropi-pette Puller P-97. The tip of the pipettes is fire-polished using a Narishige Micro Forge MF-830 under 100× magnification (e.g., Nikon MPlan 100/0.80 ELWD 210/0 lens). When filled with Csmethanesulfonate-based solutions, the resistance of such pipettes is $11-13 \mathrm{M} \Omega$. We normally use only freshly pulled pipettes made on the day of recording. To record monovalent CatSper currents in the whole-cell mode, pipettes can be filled with (in 
$\mathrm{m} M$ ): 130 Cs-methanesulfonate, 70 HEPES, 3 EGTA, 2 EDTA, 0.5 Tris-HCl, pH 7.4 adjusted with $\mathrm{CsOH}$, osmolarity $330 \mathrm{mOsm} / \mathrm{l}$. When filled with this solution, pipettes have resistance of 11-17 $\mathrm{M} \Omega$. Pipettes can also be filled with other solutions depending on the application. For sperm patch clamp, as for whole-cell patch-clamp recording from other cell types, osmolarity of the pipette solution should be about $10 \%$ higher than the bath solution. Higher tonicity of the pipette solution helps to keep the access resistance into the spermatozoon as low as possible during patch-clamp recording.

2. Before patch-clamp recording, spermatozoa are sedimented onto $5 \mathrm{~mm}$ round coverslips (WPI, Inc., cat. \# 502040). Four coverslips are placed into a single well of a Nunc 4-well plate (cat. \# 179820) and covered with $800 \mu \mathrm{l}$ of HS solution. Concentrated suspensions of mouse or human spermatozoa from the storage tube (20-50 $\mu 1$ depending on the density of the storage suspension) are gently resuspended into $800 \mu \mathrm{l}$ of HS solution above the coverslip. Coverslips are prevented from floating by taping pushing them down to the bottom of the well with the plastic tip of the pipettor. Although spermatozoa are motile, many spermatozoa will accumulate at the bottom of the well where their heads will attach to the coverslip, while flagella will vigorously beat in the solution just above the coverslip. Allow sperm cells to sediment onto the coverslips for about $20 \mathrm{~min}$ at room temperature.

3. One coverslip with spermatozoa is taken from the well and placed into a recording chamber (Warner Instruments, cat. \# RC-24E) containing HS solution. It is often useful to turn on HS perfusion to remove debris and excess spermatozoa from the recording chamber, which will increase the probability that the pipette is clean when it reaches the sperm. For capacitated sperm, one must avoid turning on perfusion immediately after a fresh coverslip is placed into the perfusion chamber to ensure that the largest number of capacitated spermatozoa is available. After the appropriate cell is chosen and patched, the perfusion can be turned on.

4. Selection of spermatozoon for patch-clamp recording. A spermatozoon should first of all have a CD. When testicular mouse spermatozoa enter the epididymis, their CDs are located in the neck region, close to the sperm head. As they travel through the epididymis, their CDs move along the midpiece and eventually arrive to the connection between midpiece and principal piece (the annulus) when spermatozoa reach the cauda epididymis. Therefore, in spermatozoa isolated from corpus epididymis, the CD is usually located close to the center of the mid-piece, while in caudal cells the CD can usually be found close to the annulus (Fig. 4.1C). In contrast to mouse spermatozoa, the human CD does not change its position during sperm movement through the epididymis and is always located in the neck region (Figs. 4.1D and 4.2A). Importantly, the CD should be oval and have a slightly elongated, spindle-like shape. CDs that appear very round and enlarged are often very fragile. We also often select sperm that are motile with the head of the spermatozoon attached to the coverslip so that the head is trapped but the CD and the rest of the flagellum continues to beat. The head should be loosely attached to the coverslip so that it rotates a little as the flagellum moves from one side to another. The loose attachment is important, since after formation of the gigaohm seal and before the transition into the whole-cell mode, the spermatozoon has to be lifted from the coverslip into the solution (see below).

5. After selection of a proper spermatozoon, the gigaohm seal is formed between the patch pipette and the spermatozoon. The micro-pipette is filled with the pipette solution and loaded into the pipette holder. Positive pressure is applied into the 
pipette to insure that the pipette solution flows out of the tip of the pipette after the pipette is immersed into the bath solution in order to keep the tip clean from any debris. Only very moderate positive pressure is usually required; however, insufficient pressure within the pipette may result in the bath solution entering the pipette, resulting in the clogging or contamination of the pipette's tip. The pipette is then brought into the bath solution.

6. As described in the Section 2, we use a $60 \times$ objective and $10 \times$ eyepieces. After the spermatozoon and the pipette are brought close to each other, we engage the additional 1.6 internal magnification of the Olympus IX71, effectively bringing the magnification of the objective to 96x. The pipette is then positioned above the CD such that the opening of the tip is aligned vertically with the $\mathrm{CD}$. The tip of the pipette is then quickly lowered toward the $\mathrm{CD}$ to be on the same plane and within a few microns of the $\mathrm{CD}$. As soon as the tip of the pipette touches the $\mathrm{CD}$, negative pressure is quickly applied into the pipette to move part of droplet into the tip and form a gigaohm seal (Figs. 4.2B and 4.3A). The technique requires precision. Many electrophysiologists working with larger cells use prolonged suction to establish the seal formation. This is possible due to the large amount of membrane in such cells, but it does not work well with human sperm cells. Usually, the only successful gigaohm seal formation between human sperm $\mathrm{CD}$ and the pipette tip is one that forms immediately. However, seal formation with mouse CDs, which are larger than human sperm CDs, requires a longer time and prolonged suction can be beneficial. In this case, after initial contact with the pipette's opening, the negative pressure in the pipette can be maintained up to a minute to gradually achieve a gigaohm seal.

7. After formation of the gigaohm seal $(>20 \mathrm{G} \Omega$ ), the spermatozoon has to be lifted from the coverslip into the solution. This is very important since the $C D$ is very small $(1-3 \mu M)$, and if the spermatozoon is attached to the coverslip, even a slight drift of the pipette during the experiment can cause disruption of the $\mathrm{CD}$ and the loss of gigaohm seal. After the spermatozoon is lifted above the coverslip, its flagellum normally continues to beat, while the spermatozoon is attached only to the pipette.

8. Before transitioning into the whole-cell mode, the stray capacitance transients are compensated with the corresponding controls of the Axo-patch 200B amplifier (to observe capacitance transients, we hold membrane potential at $0 \mathrm{mV}$ and apply 10$\mathrm{mV}$ pulses using the Membrane Test tool of PClamp 10).

9. For human spermatozoa, break-in and transition to the whole-cell mode is performed by applying short (1 ms) gradually increasing $(450-650 \mathrm{mV}, \sim 50-\mathrm{mV}$ increment) voltage pulses, sometimes combined with a light suction. Mouse spermatozoa are usually more robust and require an application of voltage ZAP (a convenient feature of Axon 200B amplifier) of the shortest duration. To apply the break-in pulses, we create a separate voltage protocol in PClamp 10. The break-in voltage pulses are applied to the spermatozoon using the high-voltage command input (rear-switched) on the back of the Axopatch 200B. The process of break-in into the spermatozoon is monitored with the Membrane Test tool of the PClamp 10 software. After application of another break-in voltage pulse, the Membrane Test tool is launched to check whether the capacitance transients reappear.

Reappearance of the capacitance transients indicates that break-in has occurred and the entire capacitance of the cell is being measured $(\sim 1 \mathrm{pF}$ for human sperm and $\sim 2.5 \mathrm{pF}$ for mouse sperm). These transients are then fitted by the Membrane Test tool to determine the capacitance of the whole sperm plasma membrane and the 
access resistance. The access resistance should be as low as possible for efficient perfusion of the interior of the spermatozoon with the pipette solution, as well as for reliable voltage clamp on the sperm plasma membrane. Access resistance is usually $25-30$ and 50-70 $\mathrm{M} \Omega$ for $\mathrm{Cs} / \mathrm{K}$-based and $N$-methyl-D-glucamine (NMDG)-based intracellular solutions, respectively.

10. After a successful break-in, the whole-cell patch-clamp experiments with spermatozoa start in exactly the same fashion as they are done with any other cell type (Fig. 4.4). If the break-in was not successful and results in nonspecific leak current (this is not a rare occasion), another appropriate sperm cell is selected and formation of the whole-cell mode of the patch-clamp technique is attempted again. An electrophysiolo-gist experienced with the sperm patch clamp forms the gigaohm seal with mouse spermatozoa in $\sim 70 \%$ of cases, and after break-in is successful in $50 \%$ of cases. With human spermatozoa, these numbers are $90 \%$ and $70 \%$.

To summarize, formation of a gigaohm seal and break-in into the spermatozoa are achieved via attaching a recording pipette to the membrane of the CD. After break-in, good intracellular electrical and perfusion access is achieved to all parts of the sperm plasma membrane and one can record ion channel activity from both sperm head and flagellum. This is evidenced by the fact that the fluorescent dye, Lucifer Yellow (MW=444 g/mol) promptly diffuses from the patch pipette throughout the whole interior of the human spermatozoa, as shown in Figs. 4.2C and 4.3B, D, and F.

\subsection{Recording from spermatozoan cell fragments}

Fragments of spermatozoa, such as head-less flagella, often reseal and their plasma membranes are intact. The fragment can be used for patch-clamp recording in order to definitively determine the localization of functional ion channels (Figs. 4.2A and D and $4.3 \mathrm{C}$ and $\mathrm{E}$ ). Beheaded and tail-less mouse sperm cells are prepared by incubating the sperm cell suspension in the presence $0.2 \mathrm{mg} / \mathrm{ml}$ of trypsin at $37^{\circ} \mathrm{C}$ for $5 \mathrm{~min}$, followed by gentle trituration with a 1-ml pipette tip. This leads to mouse sperm separation at the connection between the head and the midpiece (neck) or at the connection between the midpiece and the principal piece (annulus) (Kirichok et al., 2006; Navarro et al., 2007) (Fig. 4.3C-F). Both resulting fragments: "sperm head plus mid-piece" $(\mathrm{H}+\mathrm{M})$ and "principal piece plus midpiece" $(\mathrm{M}+\mathrm{P})$ contain the $\mathrm{CD}$ and, therefore, are suitable for patch clamp.

A simple trituration applied to human spermatozoa, without any trypsin treatment, is usually sufficient to separate some spermatozoa into head and $(\mathrm{M}+\mathrm{P})$ fragments at the neck region (Lishko et al., 2011). (M+P) contains the $\mathrm{CD}$ and can be used for whole-cell recording (Fig. 4.2A and D). Unfortunately, human spermatozoa resist the separation at the connection between the midpiece and the principal piece (annulus), and therefore, obtaining intact ( $\mathrm{H}$ $+\mathrm{M})$ fragments has not been possible.

\subsection{Compositions of recording solutions}

1. For recording of monovalent CatSper currents, the pipettes are filled with (in $\mathrm{m} M$ ): 130 Cs-methanesulfonate, 70 HEPES, 3 EGTA, 2 EDTA, 0.5 Tris-HCl, pH 7.4 adjusted with $\mathrm{CsOH}, 330 \mathrm{mOsm} / \mathrm{l}$. Bath divalent-free (DVF) solution contains (in $\mathrm{mM}$ ): 140 Cs-methanesulfonate, 40 HEPES, 1 EDTA, pH 7.4 adjusted with CsOH, $320 \mathrm{mOsm} / \mathrm{l}$. For recording of divalent CatSper currents, pipettes are filled with (in $\mathrm{m} M$ ): 145 NMDG, 0.5 Tris-Cl, 100 HEPES, and 10 BAPTA (pH 7.4 adjusted with meth-anesulfonic acid (MA)). Bath solutions for this condition contained (in $\mathrm{mM}$ ): $0-50 \mathrm{Ba}^{2+}$ or 0-50 $\mathrm{Ca}^{2+}, 150-90 \mathrm{NMDG}$, and 100 HEPES (pH 7.4, adjusted with MA). 
2. Proton currents. Pipettes are filled with (in $\mathrm{mM}$ ): 135 NMDG, 5 EGTA, and 100 HEPES or MES (pH 5.0-7.0 adjusted with MA). Bath DVF solution contains (in $\mathrm{m} M$ ): 130 NMDG, 100 HEPES or MES, 1 EDTA (pH 5.0-8.0 with MA). Proton currents tend to saturate at high positive potentials due to depletion of the intracellular $\mathrm{pH}$ buffer. To minimize the influence of this saturation, we always determine the amplitude of the sperm proton currents before intracellular proton depletion occurs (Lishko et al., 2010).

3. Potassium currents. Pipettes are filled with (in $\mathrm{mM}$ ): $130 \mathrm{KMeSO}_{3}, 4 \mathrm{KCl}, 5 \mathrm{ES} /$ HEPES, 1 EDTA, 5 EGTA (pH 6.0-7.4, adjusted with KOH). Bath solution contains (in $\mathrm{mM}$ ): $140 \mathrm{KMeSO}_{3}, 20 \mathrm{HEPES}$ (pH 7.4, adjusted with $\mathrm{KOH}$ ). To inhibit monovalent potassium current through CatSper channel $\left(\mathrm{Ca}^{2+}\right.$-selective channels conduct monovalents in the absence of other ions), $1 \mathrm{mM} \mathrm{Ca}^{2+}$ is added to the bath solution.

4. Electrophysiology experiments are usually performed at room temperature. After break-in, access resistance is usually $25-30$ and 50-70 $\mathrm{M} \Omega$ for $\mathrm{Cs} / \mathrm{K}$-based and NMDG-based intracellular solutions, respectively. Cells are often stimulated every $5 \mathrm{~s}$ with the voltage protocol; data are sampled at $2-5 \mathrm{kHz}$ and filtered at $1 \mathrm{kHz}$.

\section{POTENTIAL PITFALLS ASSOCIATED WITH SPERM PATCH CLAMP}

During whole-cell patch-clamp experiments, it is often assumed that the potential across the cell membrane is the same as applied to the pipette and that the concentrations of all ions inside the cell are the same as their concentrations in the pipette solution. This is perhaps true when whole-cell recording is performed from a relatively small round cell using a lowresistance pipette. Indeed, in this case, diffusion of the pipette solution into the cell is largely unrestricted, which helps to quickly equilibrate the intra-cellular solution with the pipette solution. Moreover, the electrical access resistance from the pipette via the cell's interior and toward any area of the membrane is low, which means very low voltage drop across this access resistance to guarantee good voltage clamp throughout the whole plasma membrane. It is difficult to achieve this ideal situation with spermatozoa.

Since spermatozoa are long (usually $>50 \mu \mathrm{m}$ ) but have small cytoplasmic cross sections $(<1$ $\mu \mathrm{m}^{2}$ throughout), diffusion from the pipette (which is usually attached to near the midpiece) to the distal parts of the flagellum is slow. For the same reason, the electrical access resistance from the pipette to these distal parts if the flagellum can be large. This may result in poor spatial voltage clamp. Problems with voltage and concentration clamp can be exacerbated by the fact that glass pipettes used for sperm patch clamp are smaller (have about three times larger resistance) due to the small size of the $\mathrm{CD}$. On the other hand, currents are usually small, kinetics slow, and ion channels are most numerous in the areas proximal to the $\mathrm{CD}$. Although we have not noticed significant problems with spatial voltage clamp during whole-cell recording from spermatozoa (as evidence by distortion of kinetics), we recommend that series resistance be kept as low as possible and sufficient time be allowed for diffusion of ions throughout the sperm.

Since the volume of the cytoplasm in the flagellum is very small, currents across the flagellar plasma membrane can significantly change the concentration of the permeant ion in the flagellum (either decrease or increase, depending on the direction of the current). This can be observed, for example, while recording $\mathrm{Ba}^{2+}$ currents through the flagellar CatSper channel in the presence of $50 \mathrm{mM} \mathrm{Ba}{ }^{2+}$ in the bath solution and no $\mathrm{Ba}^{2+}$ in the pipette solution (Kirichok et al., 2006). Although the pipette solution contained no $\mathrm{Ba}^{2+}, \mathrm{Ba}^{2+}$ accumulated inside the flagellum during the negative part of the voltage ramp and caused an outward $\mathrm{Ba}^{2+}$ current through the CatSper channel when positive potential was applied into 
the cell (Fig. 4.5). There are several steps that can be taken to improve concentration clamp in the distal flagellum. First, the osmolarity of the pipette solution should be about $10 \%$ higher than that of the bath solution to ensure that the plasma membrane is slightly expanded to maximally increase the cytosolic cross section of the flagellum. Second, between applications of voltage protocols, the membrane potential should be held as close to the reversal potential for the permeant ion as possible (to minimize the current at the holding potential). Finally, measurements of the current amplitude should be done at steady state after the test voltage step, while the intraflagellar concentration of the permeable ion is still largely unaltered.

\section{CONCLUSIONS}

By patching onto the sperm $\mathrm{CD}$, one can easily form a gigaohm seal and break-in into spermatozoa successfully. The success of the technique depends on adequate equipment that provides fine precision and stability, proper preparation and handling of the sperm cells, as well as electrophysiologist's skills and patience. The sperm patch-clamp technique opens many avenues of previously unexplored spermatozoan physiology.

\section{Acknowledgments}

This work was supported by Winkler Family Foundation Fellowship to P. L., by NIH grant U01HD045857 and a grant from the Gates Foundation to D. E. C., and by NIH grant R01HD068914 (NICHD) to Y. K. The content is solely responsibility of the authors and does not necessarily represent the official views of NICHD or the NIH.

\section{References}

Carlson AE, Quill TA, Westenbroek RE, Schuh SM, Hille B, Babcock DF. Identical phenotypes of CatSper1 and CatSper2 null sperm. The Journal of Biological Chemistry. 2005; 280:32238-32244. [PubMed: 16036917]

Carlson AE, Westenbroek RE, Quill T, Ren D, Clapham DE, Hille B, et al. CatSper1 required for evoked $\mathrm{Ca} 2+$ entry and control of flagellar function in sperm. Proceedings of the National Academy of Sciences of the United States of America. 2003; 100:14864-14868. [PubMed: 14657352]

Chung JJ, Navarro B, Krapivinsky G, Krapivinsky L, Clapham DE. A novel gene required for male fertility and functional CATSPER channel formation in spermatozoa. Nature Communications. $2011 ; 2: 153$.

Cooper TG. The epididymis, cytoplasmic droplets and male fertility. Asian Journal of Andrology. 2011; 13:130-138. [PubMed: 21076437]

Cornwall GA. New insights into epididymal biology and function. Human Reproduction Update. 2009; 15:213-227. [PubMed: 19136456]

Darszon A, Acevedo JJ, Galindo BE, Hernandez-Gonzalez EO, Nishigaki T, Trevino CL, et al. Sperm channel diversity and functional multiplicity. Reproduction. 2006; 131:977-988. [PubMed: 16735537]

Darszon A, Nishigaki T, Beltran C, Trevino CL. Calcium channels in the development, maturation, and function of spermatozoa. Physiological Reviews. 2011; 91:1305-1355. [PubMed: 22013213]

Darszon A, Wood CD, Beltran C, Sanchez D, Rodriguez E, Gorelik J, et al. Measuring ion fluxes in sperm. Methods in Cell Biology. 2004; 74:545-576. [PubMed: 15575621]

Dean J. The enigma of sperm-egg recognition in mice. Society of Reproduction and Fertility Supplement. 2007; 63:359-365. [PubMed: 17566284]

Eisenbach M, Giojalas LC. Sperm guidance in mammals-An unpaved road to the egg. Nature Reviews. Molecular Cell Biology. 2006; 7:276-285.

Florman HM, Jungnickel MK, Sutton KA. Regulating the acrosome reaction. The International Journal of Developmental Biology. 2008; 52:503-510. [PubMed: 18649263] 
Hamill OP, Marty A, Neher E, Sakmann B, Sigworth FJ. Improved patch-clamp techniques for highresolution current recording from cells and cell-free membrane patches. Pflügers Archiv. 1981; 391:85-100. [PubMed: 6270629]

Hildebrand MS, Avenarius MR, Fellous M, Zhang Y, Meyer NC, Auer J, et al. Genetic male infertility and mutation of CATSPER ion channels. European Journal of Human Genetics. 2010; 18:11781184. [PubMed: 20648059]

Hoppe PC. Fertilizing ability of mouse sperm from different epididymal regions and after washing and centrifugation. The Journal of Experimental Zoology. 1975; 192:219-222. [PubMed: 1133568]

Kaupp UB, Kashikar ND, Weyand I. Mechanisms of sperm chemotaxis. Annual Review of Physiology. 2008; 70:93-117.

Kirichok Y, Lishko PV. Rediscovering sperm ion channels with the patch-clamp technique. Molecular Human Reproduction. 2011; 17:478-499. [PubMed: 21642646]

Kirichok Y, Navarro B, Clapham DE. Whole-cell patch-clamp measurements of spermatozoa reveal an alkaline-activated Ca2+ channel. Nature. 2006; 439:737-740. [PubMed: 16467839]

Lishko PV, Botchkina IL, Fedorenko A, Kirichok Y. Acidextrusionfromhuman spermatozoa is mediated by flagellar voltage-gated proton channel. Cell. 2010; 140:327-337. [PubMed: 20144758]

Lishko PV, Botchkina IL, Kirichok Y. Progesterone activates the principal Ca2+ channel of human sperm. Nature. 2011; 471:387-391. [PubMed: 21412339]

Lishko PV, Kirichok Y. The role of Hv1 and CatSper channels in sperm activation. The Journal of Physiology. 2010; 588:4667-4672. [PubMed: 20679352]

Lishko PV, Kirichok Y, Ren D, Navarro B, Chung JJ, Clapham DE. The control of male fertility by spermatozoan ion channels. Annual Review of Physiology. 2012; 74:453-475.

Liu J, Xia J, Cho KH, Clapham DE, Ren D. CatSperbeta, a novel trans-membrane protein in the CatSper channel complex. The Journal of Biological Chemistry. 2007; 282:18945-18952. [PubMed: 17478420]

Maxwell WM, de Graaf SP, Ghaoui Rel H, Evans G. Seminal plasma effects on sperm handling and female fertility. Society of Reproduction and Fertility Supplement. 2007; 64:13-38. [PubMed: 17491139]

Meizel S. The sperm, a neuron with a tail: 'Neuronal' receptors in mammalian sperm. Biological Reviews of the Cambridge Philosophical Society. 2004; 79:713-732. [PubMed: 15682867]

Navarro B, Kirichok Y, Chung JJ, Clapham DE. Ion channels that control fertility in mammalian spermatozoa. The International Journal of Developmental Biology. 2008; 52:607-613. [PubMed: 18649274]

Navarro B, Kirichok Y, Clapham DE. KSper, a pH-sensitive K+ current that controls sperm membrane potential. Proceedings of the National Academy of Sciences of the United States of America. 2007; 104:7688-7692. [PubMed: 17460039]

Navarro B, Miki K, Clapham DE. ATP-activated P2X2 current in mouse spermatozoa. Proceedings of the National Academy of Sciences of the United States of America. 2011; 108:14342-14347. [PubMed: 21831833]

Orta G, Ferreira G, Jose O, Trevino CL, Beltran C, Darszon A. Human spermatozoa possess a calcium-dependent chloride channel that may participate in the acrosomal reaction. The Journal of Physiology. 2012; 590:2659-2675. [PubMed: 22473777]

Publicover S, Harper CV, Barratt C. [Ca2+]i signalling in sperm-Making the most of what you've got. Nature Cell Biology. 2007; 9:235-242.

Quill TA, Ren D, Clapham DE, Garbers DL. A voltage-gated ion channel expressed specifically in spermatozoa. Proceedings of the National Academy of Sciences of the United States of America 2001; 98:12527-12531. [PubMed: 11675491]

Ren D, Navarro B, Perez G, Jackson AC, Hsu S, Shi Q, et al. A sperm ion channel required for sperm motility and male fertility. Nature. 2001; 413:603-609. [PubMed: 11595941]

Ren D, Xia J. Calcium signaling through CatSper channels in mammalian fertilization. Physiology (Bethesda, Md). 2010; 25:165-175.

Sakmann B, Neher E. Patch clamp techniques for studying ionic channels in excitable membranes. Annual Review of Physiology. 1984; 46:455-472. 
Santi CM, Martinez-Lopez P, de la Vega-Beltran JL, Butler A, Alisio A, Darszon A, et al. The SLO3 sperm-specific potassium channel plays a vital role in male fertility. FEBS Letters. 2010; 584:1041-1046. [PubMed: 20138882]

Suarez SS. Control of hyperactivation in sperm. Human Reproduction Update. 2008; 14:647-657. [PubMed: 18653675]

Suarez SS, Pacey AA. Sperm transport in the female reproductive tract. Human Reproduction Update. 2006; 12:23-37. [PubMed: 16272225]

Suchyna TM, Markin VS, Sachs F. Biophysics and structure of the patch and the gigaseal. Biophysical Journal. 2009; 97:738-747. [PubMed: 19651032]

Wang H, Liu J, Cho KH, Ren D. A novel, single, transmembrane protein CATSPERG is associated with CATSPER1 channel protein. Biology of Reproduction. 2009; 81:539-544. [PubMed: 19516020]

Wassarman PM, Litscher ES. Mammalian fertilization: The egg's multifunctional zona pellucida. The International Journal of Developmental Biology. 2008; 52:665-676. [PubMed: 18649280]

Yamauchi Y, Ward MA. Preservation of ejaculated mouse spermatozoa from fertile C57BL/6 and infertile Hook1/Hook1 mice collected from the uteri of mated females. Biology of Reproduction. 2007; 76:1002-1008. [PubMed: 17314312]

Yanagimachi, R. Mammalian fertilization. In: Knobil, E.; Neill, JD., editors. The physiology of reproduction. New York: Raven Press; 1994. p. 189-317.

Zeng XH, Yang C, Kim ST, Lingle CJ, Xia XM. Deletion of the Slo3 gene abolishes alkalizationactivated $\mathrm{K}+$ current in mouse spermatozoa. Proceedings of the National Academy of Sciences of the United States of America. 2011; 108:5879-5884. [PubMed: 21427226] 

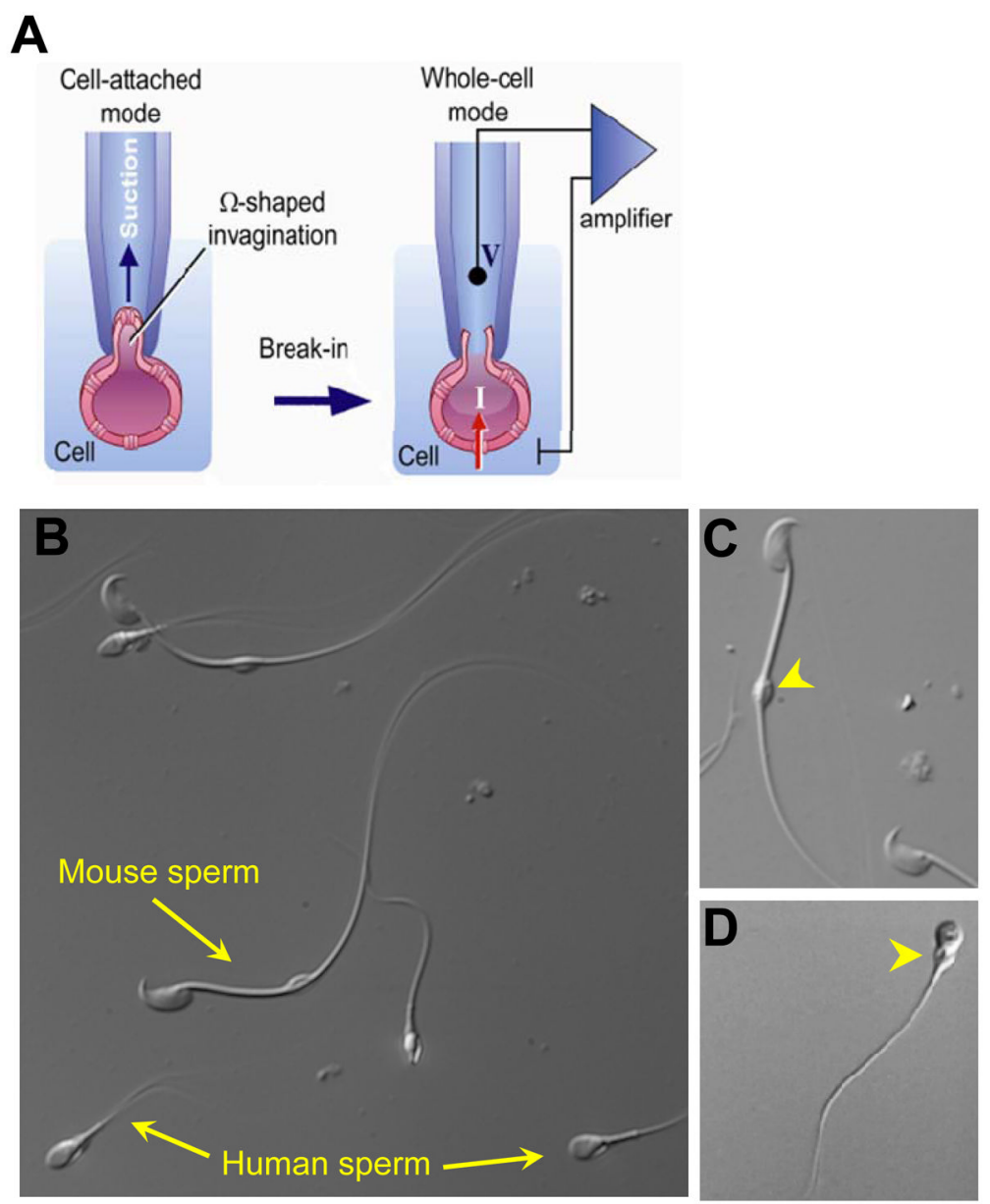

\section{Figure 4.1.}

Patching of the mammalian sperm cells. (A) To achieve successful seal formation between the pipette tip and the plasma membrane, part of the plasma membrane is gently sucked into the pipette tip. The transfer to the whole-cell mode is performed by rupturing the plasma membrane between the tip and the cell. (B) DIC image of human ejaculated sperm cells and mouse spermatozoa from cauda epididymis. (C) Mouse spermatozoon from cauda epididymis. (D) Ejaculated human spermatozoon. Cytoplasmic droplets are indicated by yellow arrowheads. Panel (A): Reproduced from Kirichok and Lishko (2011), fig. 2. Panel (C): Reproduced from Kirichok and Lishko (2011), fig. 4B. 

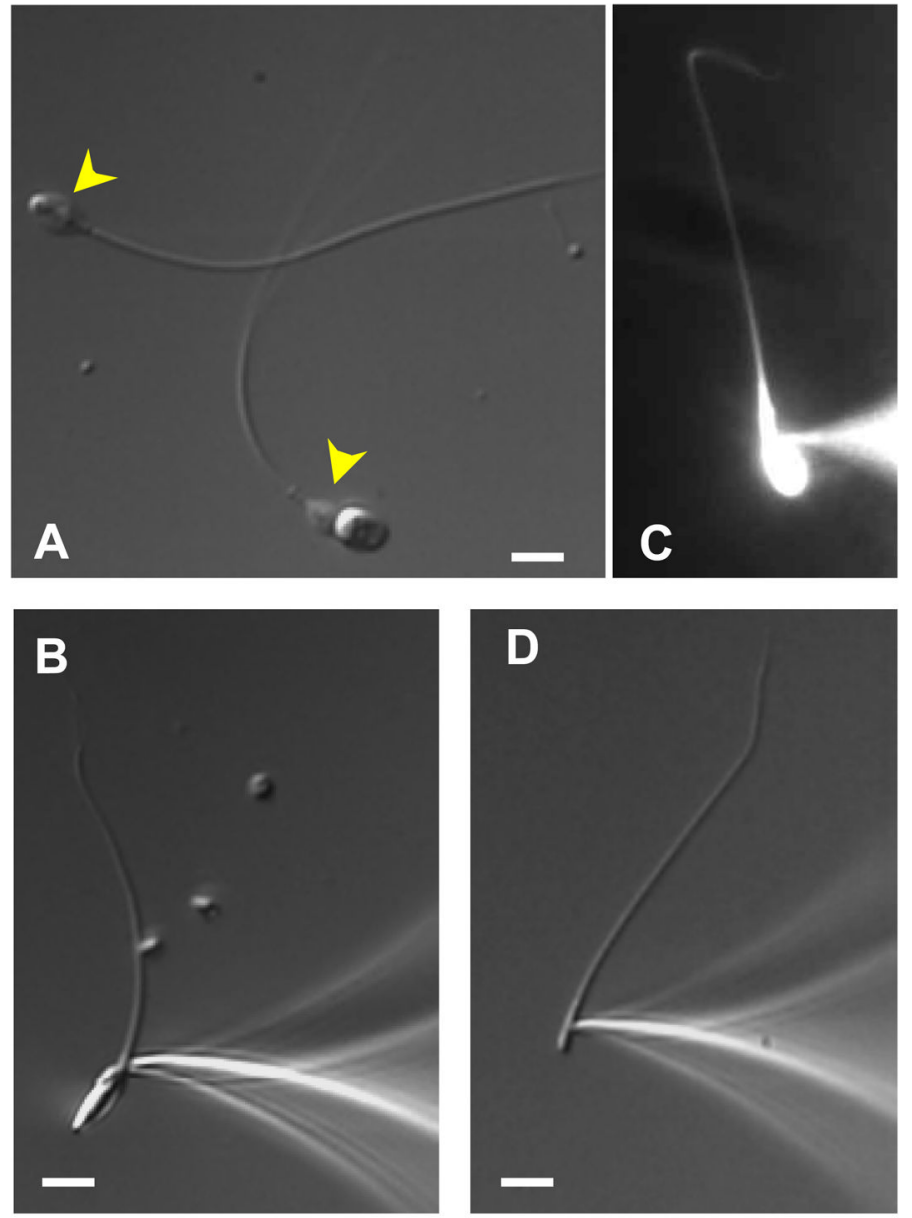

Figure 4.2.

Patch clamp of the ejaculated human spermatozoa and sperm flagella. (A) Intact human spermatozoon and the head-less sperm cell (flagellum) are shown. Cytoplasmic droplets are indicated by yellow arrowheads. (B) A tight gigaohm seal is formed with the cytoplasmic droplet of the human spermatozoon. (C) Upon break-in, the fluorescent dye Lucifer Yellow $(2 \mathrm{mM}$ ) diffuses throughout the interior of the human spermatozoon. (D) Seal formation between the pipette and human sperm flagellum. After break-in, the cytoplasmic droplet becomes inconspicuous. Scale bars, $5 \mu \mathrm{m}$. Panel (C): Reproduced from Lishko et al. (2010), fig. S1A. 

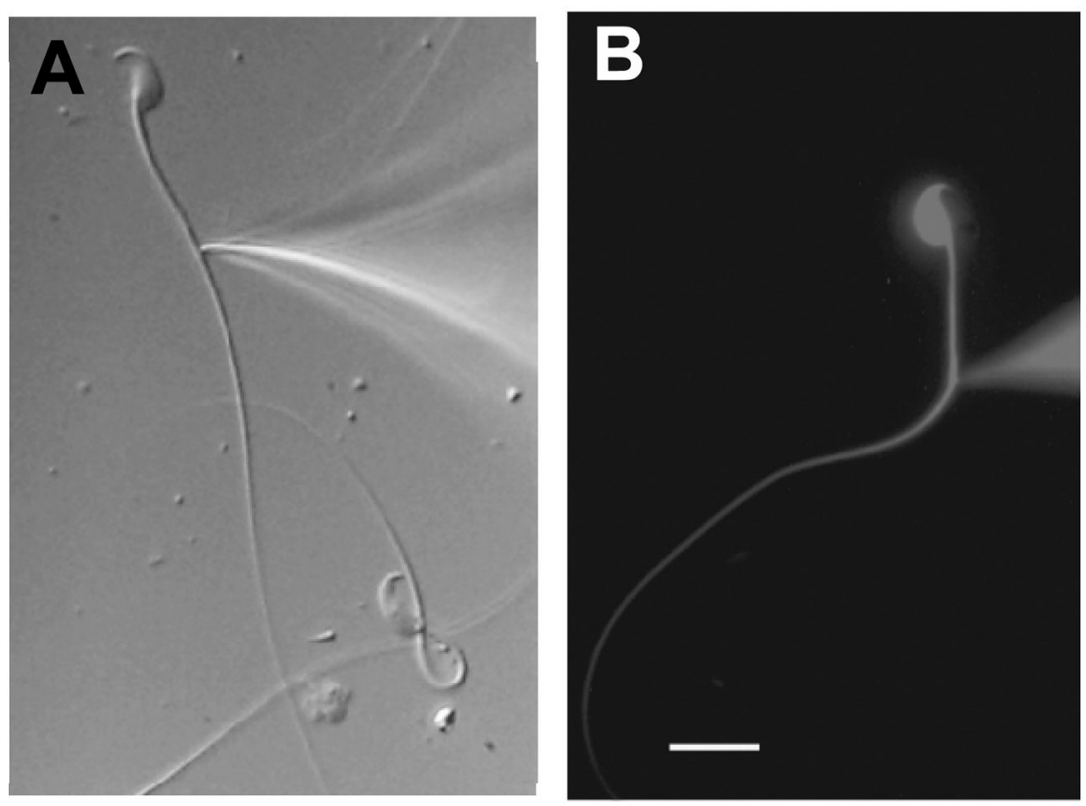

Whole sperm
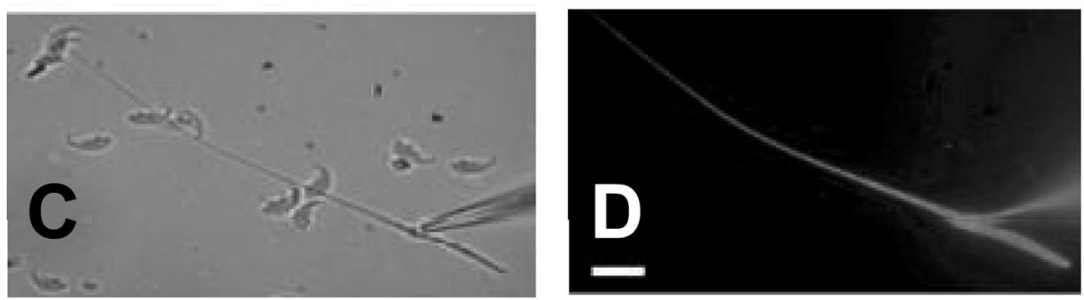

$(\mathrm{M}+\mathrm{P})$
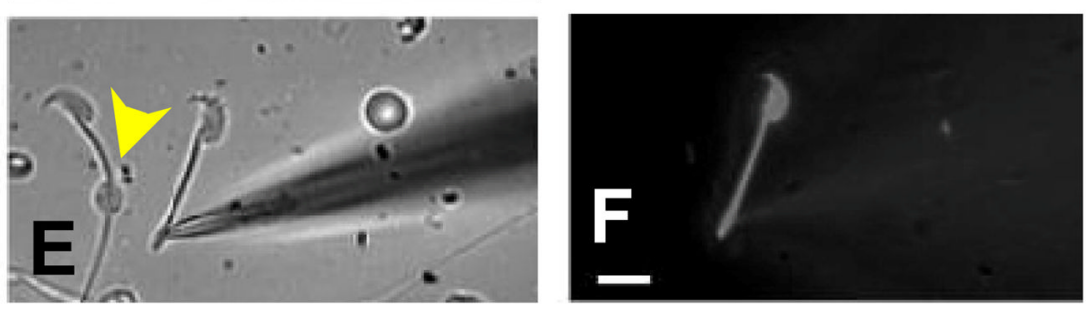

$(H+M)$

Figure 4.3.

Patch clamp of the epididymal mouse spermatozoa and their fragments. (A) Tight gigaohm seal is formed with the cytoplasmic droplet of mouse spermatozoon from cauda epididymis. (B) Lucifer Yellow $(2 \mathrm{mM})$ diffuses throughout the interior of the mouse sperm after breakin. (C-F) Fragmented mouse spermatozoa from corpus epididymis are shown. (C) Seal formation between the pipette and $(\mathrm{M}+\mathrm{P})$ fragment. $(\mathrm{M}+\mathrm{P})$ indicates midpiece+principal piece. (D) Lucifer Yellow diffuses throughout the (M+P). (E) Seal formation between the pipette and sperm head plus midpiece $(\mathrm{H}+\mathrm{M})$ fragment. The $\mathrm{CD}$ is visible (yellow arrowhead). (F) Lucifer Yellow diffuses throughout the $(\mathrm{H}+\mathrm{M})$. Scale bars, $10 \mu \mathrm{m}$. Panel (A): Reproduced from Lishko et al. (2011), fig. 1C. Panel (B): Reproduced from Kirichok et al. (2006), fig. 1A. Panel (C-F): Reproduced from Navarro et al. (2007), fig. 2D and E. 
A

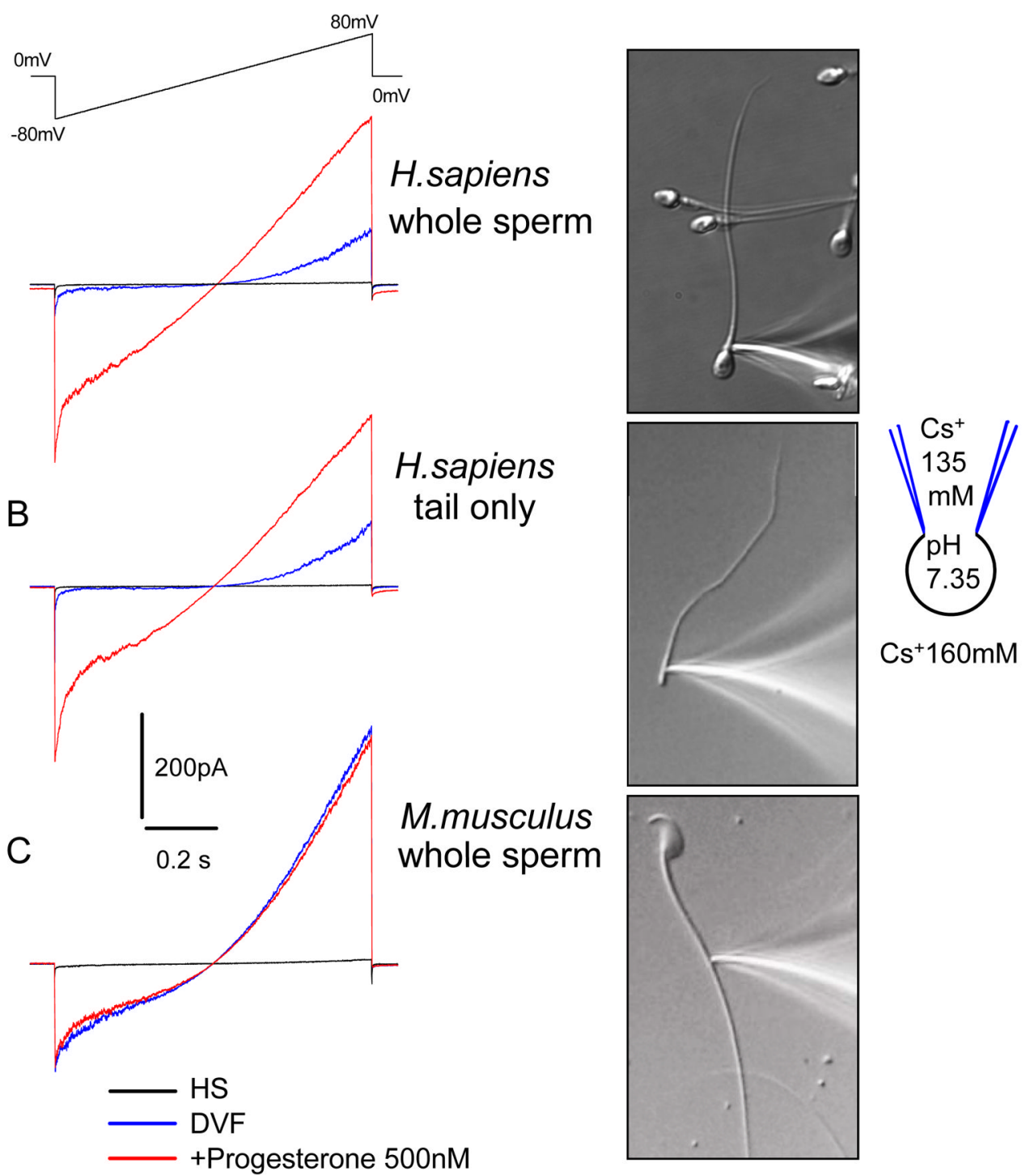

Figure 4.4.

Recording of the human and mouse CatSper currents. (A) Representative monovalent whole-cell CatSper currents recorded from human spermatozoon in the absence (blue trace) and in the presence (red trace) of $500 \mathrm{n} M$ progesterone (P). Right, the tight seal formed with the cytoplasmic droplet of the whole human spermatozoon. (B) Representative wholeflagellum human CatSper current. Right, image of the human sperm flagellum tightly attached to the recording pipette. (C) Representative, monovalent whole-cell CatSper currents recorded from mouse epididymal sperm cell in the absence (blue trace) and in the presence (red trace) of $500 \mathrm{n} M$ P. Caudal epididymal sperm cells were used. Right, image of the mouse spermatozoon isolated from cauda epididymis and tightly attached to the recording pipette. Currents were elicited by a voltage ramps from a holding potential of 0 $\mathrm{mV}$. Ramps were applied from -80 to $80 \mathrm{mV}$ in HS and divalent-free solution. Baseline currents recorded in HS solution. Reproduced from Lishko et al. (2011), fig. 1. 
Noncapacitated

Capacitated

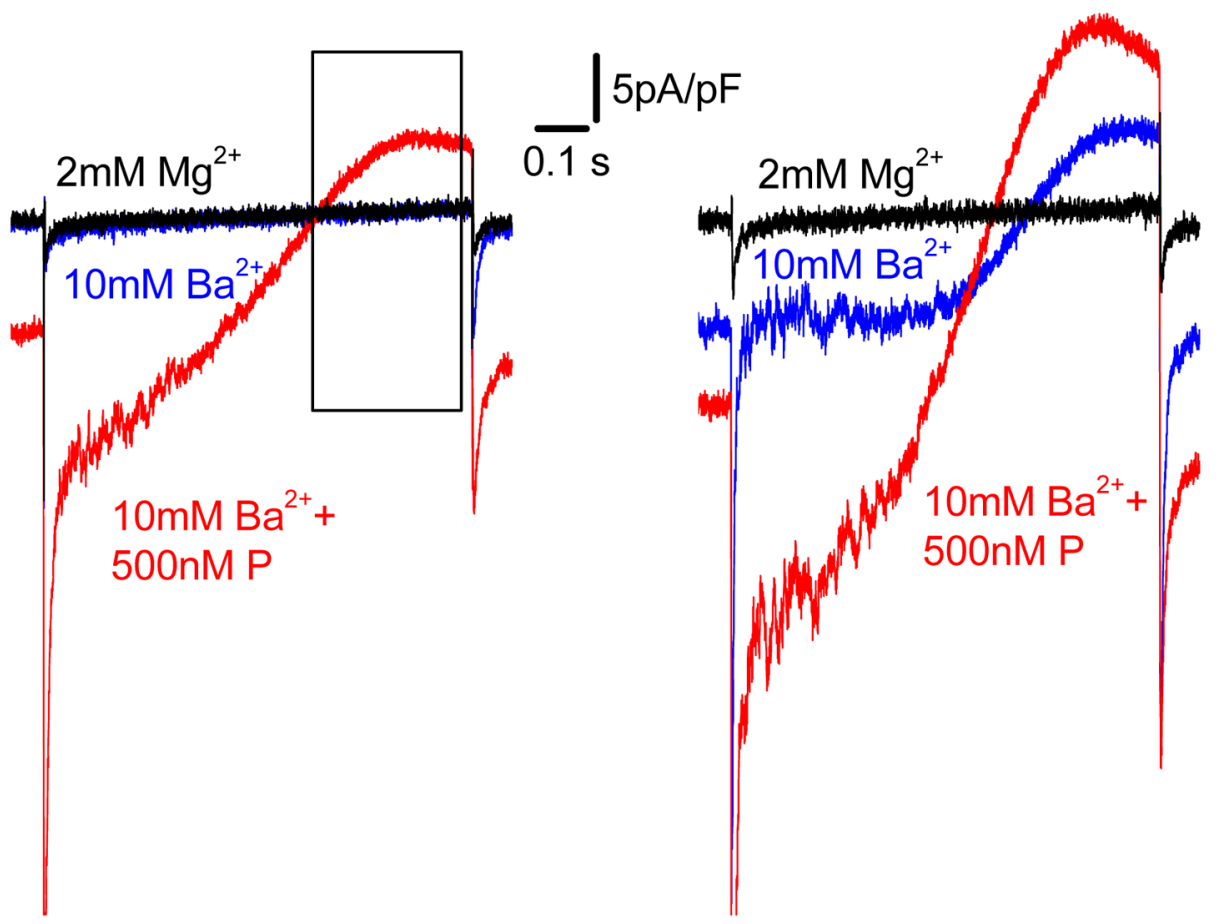

Figure 4.5.

Recording of the divalent CatSper currents from ejaculated (left panel) and capacitated (right panel) human sperm cells. Representative $\mathrm{Ba}^{2+}$ whole-cell CatSper currents recorded from human spermatozoon in the absence (blue trace) and in the presence (red trace) of 500 $\mathrm{n} M \mathrm{P}$. Currents were elicited by a voltage ramps from a holding potential of $0 \mathrm{mV}$. Ramps were applied from -80 to $+80 \mathrm{mV}$ in bath solution containing $10 \mathrm{mM} \mathrm{Ba}^{2+}$ (see Section 4.4). Baseline currents (black traces) were recorded in the same bath solution supplemented with $2 \mathrm{mM} \mathrm{MgCl} 2$. The black frame on the left panel indicates outward $\mathrm{Ba}^{2+}$ current through the CatSper channel during application of positive potentials. Although the pipette solution contained no $\mathrm{Ba}^{2+}$ ions in this case, $\mathrm{Ba}^{2+}$ does accumulate inside the flagellum during the negative part of the voltage ramp and is responsible for this outward current. 